\title{
A Generalisation of Transversals for Latin Squares
}

\author{
Ian M. Wanless \\ Christ Church \\ St Aldates \\ Oxford OX1 1DP \\ United Kingdom \\ wanless@maths.ox.ac.uk
}

We define a $k$-plex to be a partial latin square of order $n$ containing $k n$ entries such that exactly $k$ entries lie in each row and column and each of $n$ symbols occurs exactly $k$ times. A transversal of a latin square corresponds to the case $k=1$. For $k>n / 4$ we prove that not all $k$-plexes are completable to latin squares. Certain latin squares, including the Cayley tables of many groups, are shown to contain no $(2 c+1)$-plex for any integer $c$. However, Cayley tables of soluble groups have a $2 c$-plex for each possible c. We conjecture that this is true for all latin squares and confirm this for orders $n \leq 8$. Finally, we demonstrate the existence of indivisible $k$-plexes, meaning that they contain no $c$-plex for $1 \leq c<k$.

Submitted: September 5, 2001; Accepted: March 18, 2002.

MR Subject Classification: 05B15

\section{$\S 1 . \quad$ Introduction}

A partial latin square of order $n$ is a matrix of order $n$ in which each cell is either blank or contains one of $\{1,2, \ldots, n\}$ (or some other fixed set of cardinality $n$ ), and which has the property that no symbol occurs twice within any row or column. A cell which is not blank is said to be filled. A partial latin square with every cell filled is a latin square. The set of partial latin squares of order $n$ is denoted by $\operatorname{PLS}(n)$, and the set of latin squares of order $n$ by $\operatorname{LS}(n)$. We say that $P_{1} \in \operatorname{PLS}(n)$ contains $P_{2} \in \operatorname{PLS}(n)$ if every filled cell of $P_{2}$ agrees with the corresponding cell of $P_{1} . P \in \operatorname{PLS}(n)$ is said to be completable if there is some $L \in \mathrm{LS}(n)$ such that $L$ contains $P$. On the other hand, $P$ is said to be maximal if the only partial latin square which contains $P$ is $P$ itself.

We coin the name $k$-plex of order $n$ for a $K \in \operatorname{PLS}(n)$ in which each row and column of $K$ contains exactly $k$ filled cells and each symbol occurs exactly $k$ times in $K$. The entries on a transversal of a latin square form a 1-plex. In the statistical literature (eg. Finney [10-12]) a transversal is sometimes called a directrix; while the terms duplex, triplex and quadruplex are used for a 2-plex, 3-plex and 4-plex respectively. This is the motivation for our terminology, which permits a natural extension to arbitrarily large $k$. We use the plural form " $k$-plexes" (sometimes dropping the $k$ in statements 
of a general nature) rather than following the classical pattern which dictates that the plurals of duplex and triplex are duplices and triplices. Note that plexes are defined without being named in [8, p.34]. Also, some other names have been used for $k$-plexes. For example, Colbourn and Dinitz [5] use the name $k$-transversal while Burton [3] uses $k$-stagger. The former name is problematic since it has been used in other senses (see $[7, \mathrm{p} .453]$ and $[8, \mathrm{p} .23])$, besides which we feel that $k$-plex is more faithful to historical context.

There are some natural ways to combine plexes. Suppose that $K_{1}$ and $K_{2}$ are $k$-plexes of respective orders $n_{1}$ and $n_{2}$. For convenience, we assume that the symbols occurring in $K_{1}$ are disjoint from those in $K_{2}$ (if not, label each symbol with a subscript denoting which $k$-plex it belongs to). Then the direct sum of $K_{1}$ and $K_{2}$ is defined by

$$
K_{1} \oplus K_{2}=\left(\begin{array}{cc}
K_{1} & \emptyset_{1} \\
\emptyset_{2} & K_{2}
\end{array}\right),
$$

where $\emptyset_{1}$ and $\emptyset_{2}$ respectively denote $n_{1} \times n_{2}$ and $n_{2} \times n_{1}$ blocks in which every cell is empty. It should be obvious that $K_{1} \oplus K_{2}$ is a $k$-plex of order $n_{1}+n_{2}$.

Following Finney [12], we say that two plexes in the same square are parallel if they have no filled cells in common. The union of an $a$-plex and a parallel $b$-plex of a latin square $L$ is an $(a+b)$-plex of $L$. However it is not in general possible to split an $(a+b)$-plex into an $a$-plex and a parallel $b$-plex. Consider for example a duplex which consists of $\frac{1}{2} n$ disjoint intercalates (latin subsquares of order 2). Such a duplex does not contain a partial transversal of length more than $\frac{1}{2} n$, so it is a long way from containing a 1-plex. Another example can be found in (4) below, which shows a 3-plex which contains no 1-plex, and some more general examples are discussed in $\S 5$.

The entries not included in a $k$-plex of a latin square $L$ of order $n$ form a $(n-k)$-plex of $L$. Together the $k$-plex and its complementary $(n-k)$-plex are an example of what is called an orthogonal partition of $L$. More generally, if $L$ is decomposed into disjoint parts $K_{1}, K_{2}, \ldots, K_{d}$ where $K_{i}$ is a $k_{i}$-plex then we call this a $\left(k_{1}, k_{2}, \ldots, k_{d}\right)$-partition of $L$. The notation $\left(k_{1}^{\alpha_{1}}, k_{2}^{\alpha_{2}}, \ldots\right)$ can be used as shorthand for a partition with $\alpha_{1}$ parts which are $k_{1}$-plexes, $\alpha_{2}$ parts which are $k_{2}$-plexes, etc. A case of particular interest is when all parts are the same size. We call a $\left(k^{n / k}\right)$-partition a $k$-partition.

The concept of orthogonal partitions in latin squares goes at least as far back as Finney [10]. The idea is discussed in a more general setting by Gilliland [16] and Bailey [2]. Orthogonal partitions are also related to orthogonal frequency squares as discussed, for example, in [5] and [8].

Two latin squares are said to be isotopic if one can be obtained from the other by permuting the rows, columns and symbols. The main class of a latin square $L$ is the set of squares which can be obtained from $L$ by isotopies, and also by permuting the roles played by rows, columns and symbols. The definition of $k$-plex implies that, for each $k$, the number of $k$-plexes is a main class invariant. Indeed, this is true more generally for other types of orthogonal partitions, which is one of the reasons why they are an 
interesting concept. We will discuss the utility of plexes for distinguishing main classes in $\S 4$.

In the next section we deal with the question of which plexes are completable to latin squares. Following that is a section on the existence of plexes in Cayley tables of groups. Section 4 looks at plexes in latin squares of orders up to 8 and the last section before the final summary proves that some plexes cannot be split into non-trivial subplexes.

\section{$\S 2 . \quad$ Completability of plexes}

It is easily established that there is no completable 1-plex of order 2. This is just a small order anomaly, as shown by our first result.

Theorem 1. If $n>2$ then there exists $L \in \operatorname{LS}(n)$ which contains a $k$-plex for each $k$ satisfying $0 \leq k \leq n$.

Proof: If $n>2$ and $n \neq 6$, a celebrated result says that there are two orthogonal latin squares of order $n$. A square with an orthogonal mate has a 1-partition and the union of any $k$ of these parts gives us a $k$-plex.

For $n=6$ there is no pair of orthogonal squares, however we can get close enough for present purposes. Finney [10] found the following example from $\operatorname{LS}(6)$ which contains 4 parallel transversals.

$$
\left(\begin{array}{llllll}
1_{a} & 2 & 3_{b} & 4_{c} & 5 & 6_{d} \\
2_{c} & 1_{d} & 6 & 5_{b} & 4_{a} & 3 \\
3 & 4_{b} & 1 & 2_{d} & 6_{c} & 5_{a} \\
4 & 6_{a} & 5_{c} & 1 & 3_{d} & 2_{b} \\
5_{d} & 3_{c} & 2_{a} & 6 & 1_{b} & 4 \\
6_{b} & 5 & 4_{d} & 3_{a} & 2 & 1_{c}
\end{array}\right)
$$

The four separate transversals are indicated by the subscripts $a, b, c$ and $d$. The symbols without a subscript form a 2-plex which together with $k-2$ of the transversals yields a $k$-plex for $k=2,3,4,5,6$. The four marked transversals supply examples for $k=1$. $\odot$

A corollary of the result just proved is that if $0 \leq k \leq n$ and $n>2$ then there is a completable $k$-plex of order $n$. However, our next result shows that not all $k$-plexes are completable.

Theorem 2. If $1<k<n$ and $k>\frac{1}{4} n$ there exists an uncompletable $k$-plex of order $n$.

Proof: Our construction divides into three cases.

Case 1. $\frac{1}{4} n<k \leq \frac{1}{2} n$.

Let $m=\left\lfloor\frac{1}{2} n\right\rfloor$. Let $K=A \oplus D \in \operatorname{PLS}(n)$,

$$
K=\left(\begin{array}{ll}
A & B \\
C & D
\end{array}\right)
$$


where $A$ is a $k$-plex of order $m$ on the symbols $\{1,2, \ldots, m\}, D$ is a $k$-plex of order $n-m$ on the symbols $\{m+1, m+2, \ldots, n\}$, and both $B$ and $C$ are empty. Suppose that $K$ has a completion to $L \in \operatorname{LS}(n)$, and let $B^{\prime}$ be the submatrix of $L$ corresponding to $B$. We count the number of occurrences of each symbol in $B^{\prime}$. The symbols $\{1,2, \ldots, m\}$ already occur $k$ times in $A$ within the first $m$ rows of $K$, so they can occur at most $m-k$ times each in $B^{\prime}$. Similarly the symbols $\{m+1, m+2, \ldots, n\}$ already occur $k$ times in $D$ within the last $n-m$ columns of $K$, so they can occur at most $n-m-k$ times each in $B^{\prime}$. But $B^{\prime}$ has $m(n-m)$ cells, so it must be that

$$
\begin{aligned}
0 & \leq m(m-k)+(n-m)(n-m-k)-m(n-m)=3\left\lfloor\frac{1}{2} n\right\rfloor\left(\left\lfloor\frac{1}{2} n\right\rfloor-n\right)+n^{2}-k n \\
& \leq 3\left(\frac{1}{4}-\frac{1}{4} n^{2}\right)+n^{2}-\frac{1}{4}(n+1) n=\frac{1}{4}(3-n)
\end{aligned}
$$

which means $n \leq 3$. However, this contradicts $2 \leq k \leq \frac{1}{2} n$ so we conclude that $K$ is not completable.

Case 2. $\frac{1}{2} n<k<n$ and $n$ is even.

Let $m=\frac{1}{2} n$ and $j=k-m$. By Theorem 1 we can find $M \in \operatorname{LS}(m)$ which uses the symbols $\{1,2, \ldots, m\}$ and which is the disjoint union of a $j$-plex $J$ and an $(m-j)$-plex which we denote $M \backslash J$. Let $K \in \operatorname{PLS}(n)$ be composed of four $m \times m$ blocks thus:

$$
\left(\begin{array}{ll}
A & B \\
C & D
\end{array}\right)
$$

where $A$ is a copy of $M, B=C$ is a copy of $J$ with each symbol increased by $m$ and $D$ is a copy of $M$ in which each of the cells in $M \backslash J$ has had its symbol increased by $m$.

Notice that each of the symbols $\{1,2, \ldots, m\}$ occurs within every row of $A$, while each of the symbols $\{m+1, m+2, \ldots, 2 m=n\}$ occurs in each of the last $m$ columns of $K$. Hence none of the blank cells in $B$ can be filled without breaching the latin property. A similar argument applies to the only other blank cells in $K$, namely those in $C$. It is apparent then that $K$ is maximal, in which case it is certainly not completable. (In fact this construction is a special case of one for maximal partial latin squares given by Horák and Rosa [18].)

Case 3. $\frac{1}{2} n<k<n$ and $n$ is odd.

We index our rows, columns and symbols with the congruence classes of integers modulo $n$. Let $K^{\prime}$ be the order $n$ matrix defined by $K_{i j}^{\prime} \equiv i+j$ if $j-i \in\{0,1, \ldots k-1\}$ with $K_{i j}^{\prime}$ not being filled otherwise. Thus $K^{\prime}$ consists of $k$ broken diagonals from a cyclic square of order $n$. As $n$ is odd, each of these diagonals is a 1-plex and $K^{\prime}$ is a $k$-plex. Now form $K$ as follows

$$
K_{i j}= \begin{cases}0 & \text { if } i=1, j=0 \\ k+1 & \text { if } i=0, j=k \\ \text { unfilled } & \text { if } i=j=0 \\ \text { unfilled } & \text { if } i=1, j=k \\ K_{i j}^{\prime} & \text { otherwise }\end{cases}
$$


So $K$ is constructed from $K^{\prime}$ by performing the following surgery on rows 0 and 1 :

$$
\left(\begin{array}{cccccc}
0 & 1 & 2 & \ldots & k-1 & \uparrow \\
\downarrow & 2 & 3 & \ldots & k & k+1
\end{array}\right)
$$

Note that the cells $K_{10}^{\prime}$ and $K_{0 k}^{\prime}$ are necessarily empty since $k<n$. It is clear then, that our surgery preserves the number of filled cells in each row and column and the number of occurrences of each symbol. Also $K$ must be latin since we are either swapping the zeroes in rows 0 and 1 (in the case $k=n-1$ ) or else 0 does not occur in row 1 of $K^{\prime}$, nor does $k+1$ occur in row 0 . So $K$, like $K^{\prime}$, is a $k$-plex. However, while $K^{\prime}$ is clearly completable to a cyclic square, we claim that $K$ is not completable.

The symbols $1,2, \ldots k-1$ already occur in row 0 , while symbols $n-k+1, n-k+$ $2, \ldots, n-1,0$ occur in column 0 . But $n-k+1 \leq k$ because $2 k>n$, so this leaves no symbol available to fill cell 00 .

This completes the case, and the proof of the theorem.

Theorem 2 provides a partial answer to a problem posed by Donovan [9, p.255], who asked for which $n$ and $k$ does there exist a non-completable $k$-plex of order $n$. It has been suggested by several people (eg Burton [3], and Daykin and Häggkvist [6]) that Theorem 2 encompasses the whole answer to this question. In other words, they conjecture that if $k \leq \frac{1}{4} n$ then every $k$-plex is completable. It seems certain that for $k<<n$ every $k$-plex is completable. This has already been proved when $n \equiv 0 \bmod 16$ in [6]. Also, an as yet unpublished result of Chetwynd and Häggkvist [4] shows the result for all even $n$. The following partial extension result due to Burton [3] is also relevant.

Theorem 3. For $k \leq \frac{1}{4} n$ every $k$-plex of order $n$ is contained in a $(k+1)$-plex of order $n$.

Proof: Let $K$ be the $k$-plex to be extended. By direct application of Hall's condition it can be seen that any $n$ sets, each with at least $m$ elements chosen from $\{1,2, \ldots, n\}$ have a system of distinct representatives if $m \geq \frac{1}{2} n$ and each symbol occurs at least $m$ times among the $n$ sets. We use this result twice. Firstly, for each row we select a column which is not used in that row in $K$, with the columns chosen being distinct for different rows. Once we have chosen the positions we apply the result again to choose the symbols occupying those positions. In each position there are at least $n-2 k \geq \frac{1}{2} n$ symbols which can be used, so we can find a 1-plex which can be used to extend $K$ to a $(k+1)$-plex.

We caution that the plexes in Theorem 3 are not situated in a particular latin square; conceivably they are not completable at all. We shall meet in the next section plenty of latin squares which have $k$-plexes which are not included in $(k+1)$-plexes of the same square, even for $k<<n$. Then in $\S 5$ we will see that no converse of Theorem 3 is possible. 


\section{$\S 3 . \quad$ Cayley tables of groups}

Let $G=\left\{g_{1}, g_{2}, \ldots, g_{n}\right\}$ be a finite group. The Cayley table for (this enumeration of) $G$ is the matrix of order $n$ whose entry in row $r$ and column $c$ is $g_{r} g_{c}$. The existence of inverses in $G$ guarantees that any Cayley table of $G$ is a latin square. In this section we examine the existence question for plexes in Cayley tables. We say that a group possesses a $k$-plex if its Cayley table does. We first dispose of one easy case. It is well known (see below or [7]) that if the Cayley table of a group has a transversal then it has a 1-partition. We can simply take the union of $k$ of these transversals, to yield:

Theorem 4. If the Cayley table of a group possesses a transversal then it has a 1 -partition and hence a $k$-plex for every possible $k$.

"Translation" using the group's multiplication operation is the key to finding parallel transversals, once one has been found. Suppose we know of a transversal that comprises a choice from each row $i$ of an element $g_{i}$. Let $g$ be any fixed element of $G$. Then if we select from each row $i$ the element $g_{i} g$ this will give a new transversal and as $g$ ranges over $G$ the transversals so produced will be mutually parallel and thus provide a 1-partition of $L$. The crucial point is that this translation respects collinearity in columns in the following sense. If $g_{i}$ and $g_{j}$ are two entries which get translated to, respectively, $g_{i} g$ and $g_{j} g$ then $g_{i} g$ is in the same column as $g_{j} g$ if and only if $g_{i}$ was in the same column as $g_{j}$. It follows that if we have any $k$-plex and translate it by a fixed group element we get another $k$-plex. However, if $k>1$ the new $k$-plex need not be parallel to the original one. As an example, consider the following Cayley table for $S_{3}$ in which the six translates of a duplex $D$ (which is marked in bold) are shown. No three of these six translates decompose the table, although some pairs of translates are parallel.

$$
\left(\begin{array}{llllll}
1_{2,5} & 2_{3,6} & \mathbf{3}_{1,4} & 4_{3,6} & \mathbf{5}_{1,4} & 6_{2,5} \\
2_{4,6} & 3_{4,5} & 1_{5,6} & \boldsymbol{6}_{1,2} & 4_{2,3} & \mathbf{5}_{1,3} \\
\mathbf{3}_{1,3} & \mathbf{1}_{1,2} & 2_{2,3} & 5_{4,5} & 6_{5,6} & 4_{4,6} \\
\boldsymbol{4}_{1,2} & 5_{2,3} & \boldsymbol{6}_{1,3} & 1_{4,6} & 2_{4,5} & 3_{5,6} \\
5_{5,6} & 6_{4,6} & 4_{4,5} & 3_{2,3} & \mathbf{1}_{1,3} & \mathbf{2}_{1,2} \\
6_{3,4} & \mathbf{4}_{1,5} & 5_{2,6} & \mathbf{2}_{1,5} & 3_{2,6} & 1_{3,4}
\end{array}\right)
$$

The interpretation of this Cayley table is as follows. The mapping

$$
\begin{aligned}
& (1)(2)(3) \rightarrow 1, \quad(123) \rightarrow 2, \quad(132) \rightarrow 3, \\
& (12)(3) \rightarrow 4, \quad(13)(2) \rightarrow 5, \quad(1)(23) \rightarrow 6,
\end{aligned}
$$

has been chosen for $S_{3}$ and permutations act on the left. The subscripts on the entries indicate which translates of $D$ they are included in. $D$ itself consists of the 12 entries 
which have 1 among their subscripts, denoting translation by the identity. The duplex obtained by translating $D$ by the group element (123) consists of entries with subscript 2 , etc. Note that the intersection between translates can be empty (eg, translates 1 and 6 ) but is usually non-trivial (eg, translates 1 and 3 overlap in four places).

Hall and Paige [17] proved that a finite soluble group $G$ possesses a transversal if and only if the Sylow 2-subgroups of $G$ are trivial or non-cyclic. They conjectured that the stipulation that $G$ is soluble can be dropped, in which case it would follow that all non-soluble groups have a transversal. By Theorem 4 then, the only case that would remain for us would be groups with a non-trivial cyclic Sylow 2-subgroup. We shall resolve the existence question for plexes in that case below, but first we need some preliminary results. The first was noted by Hall and Paige [17], and also appears in $[7$, p.36].

Theorem 5. If $G$ is a finite group with a non-trivial cyclic Sylow 2-subgroup $S$ then $G$ has a normal subgroup $K$ of odd order such that $S$ is a set of coset representatives for $K$.

A latin square of order $m q$ is said to be of $q$-step type if it can be represented by a matrix of $q \times q$ blocks $A_{i j}$ as follows

$$
\left(\begin{array}{cccc}
A_{11} & A_{12} & \cdots & A_{1 m} \\
A_{21} & A_{22} & \cdots & A_{2 m} \\
\vdots & \vdots & \ddots & \vdots \\
A_{m 1} & A_{m 2} & \cdots & A_{m m}
\end{array}\right)
$$

where each block $A_{i j}$ is a latin subsquare of order $q$ and two blocks $A_{i j}$ and $A_{i^{\prime} j^{\prime}}$ contain the same symbols if and only if $i+j \equiv i^{\prime}+j^{\prime} \bmod m$.

Theorem 6. Suppose that $q$ and $k$ are odd integers and $m$ is even. No q-step type latin square of order $m q$ possesses a $k$-plex.

Proof: Our proof is based on that of Theorem 12.3.1 in [7], which treats the case $k=1$. Suppose that $L$ is a $q$-step type latin square of order $n=m q$, as shown in (1). By relabelling if necessary we may assume that $L$ uses the symbols $\{1,2, \ldots, n\}$ and that each subsquare $A_{i j}$ contains the symbols $\{h q-q+1, h q-q+2, \ldots, h q-1, h q\}$ where $h \equiv i+j \bmod m$.

Let us suppose that the theorem fails and that $K$ is a $k$-plex of $L$. We arbitrarily fix an order of the $k n$ entries in $K$. For $i=1,2, \ldots, k n$ let the $i$-th entry in $K$ be the symbol $a_{i} q-b_{i}\left(\right.$ where $\left.0 \leq b_{i}<q\right)$ chosen from the block $A_{c_{i} d_{i}}$. Note that $a_{i} \equiv c_{i}+d_{i} \bmod m$ by the way we chose our labelling.

Now each symbol occurs $k$ times in $K$, and for a fixed $j \in\{1,2, \ldots, m\}$ there are $q$ integers in the range $\{1,2, \ldots, n\}$ of the form $j q-b$ where $0 \leq b<q$. Hence if we add 
$a_{i}$ over the entries of $K$ we get

$$
\sum_{i=1}^{k n} a_{i}=k q \sum_{j=1}^{m} j=\frac{1}{2} k q m(m+1)
$$

Fix any $j \in\{1,2, \ldots, m\}$. The $k$-plex $K$ must contain exactly $k q$ entries from the $q$ columns of $L$ represented by the blocks $A_{1 j}, A_{2 j}, \ldots, A_{m j}$. Thus there are $k q$ indices for which $d_{i}$ takes the value $j$. Likewise, there are $k q$ entries from the $q$ rows of $L$ represented by the blocks $A_{j 1}, A_{j 2}, \ldots, A_{j m}$, so there are $k q$ indices for which $c_{i}$ takes the value $j$. Thus, modulo $m$,

$$
\sum_{i=1}^{k n} a_{i} \equiv \sum_{i=1}^{k n} c_{i}+\sum_{i=1}^{k n} d_{i}=2 k q \sum_{j=1}^{m} j=k q m(m+1) \equiv 0
$$

Combining this result with (2) we deduce that 2 divides $k q(m+1)$ which contradicts the parities chosen for $k, q$ and $m$. We conclude that $L$ has no $k$-plex.

Theorem 6 shows that some latin squares do not contain a $k$-plex for any odd $k$. A particularly important special case of this result is included in our next theorem.

Theorem 7. Let $G$ be a group of finite order $n$ with a non-trivial cyclic Sylow 2subgroup. The Cayley table of $G$ contains no $k$-plex for any odd $k$ but has a 2-partition and hence contains a $k$-plex for every even $k$ in the range $0 \leq k \leq n$.

Let $n=m q$ where $q$ is odd and $m \geq 2$ is a power of 2 . Let $S=\langle s\rangle$ be a Sylow 2-subgroup of $G$. By Theorem 5 there exists a normal subgroup $N$ of $G$ such that $S$ is a set of coset representatives for $N$. Note that $N=\left\{g_{1}, g_{2}, \ldots, g_{q}\right\}$ has order $q$ and $S$ has order $m$. We can order the elements of $G$ as follows:

$$
\left\{g_{1}, g_{2}, \ldots, g_{q}, s g_{1}, s g_{2}, \ldots, s g_{q}, s^{2} g_{1}, s^{2} g_{2}, \ldots, s^{2} g_{q}, \ldots, s^{m-1} g_{1}, s^{m-1} g_{2}, \ldots, s^{m-1} g_{q}\right\}
$$

Using this enumeration for $G$ we build a Cayley table, the body of which is a latin square $L$. We claim that $L$ is of $q$-step type. To see this, break $L$ up into blocks $A_{i j}$ as follows

$$
\left(\begin{array}{cccc}
A_{00} & A_{01} & \cdots & A_{0, m-1} \\
A_{10} & A_{11} & \cdots & A_{1, m-1} \\
\vdots & \vdots & \ddots & \vdots \\
A_{m-1,0} & A_{m-1,1} & \cdots & A_{m-1, m-1}
\end{array}\right) .
$$

By the chosen enumeration, every element in $A_{i j}$ belongs to the set $s^{i} N s^{j} N$, which is just $s^{i+j} N$ since $N$ is normal. Since $s^{i+j} N$ consists of only $q$ symbols and $A_{i j}$ is a block of order $q$ we see that $A_{i j}$ must in fact be a latin subsquare of $L$ and the remaining requirement in the definition of $q$-step type is immediate from the fact that $S=\langle s\rangle$ is a 
set of coset representatives for $N$. At this point we can apply Theorem 6 and rule out $L$ having a $k$-plex for any odd $k$.

The existence of a $k$-plex in a Cayley table of $G$ is invariant with the enumeration chosen for $G$, so to complete the proof of the theorem it suffices to exhibit a 2-partition of $L$. We do this by first arguing that each of the subsquares $A_{i j}$ has a 1-partition. For $A_{11}$ this follows from Theorem 4 since $A_{11}$ is a Cayley table for $N$, which is a group of odd order (the main diagonal is always a transversal of the Cayley table of a group of odd order). Also, for any given $i$ and $j$, the subsquare $A_{i j}$ can be obtained from $A_{11}$ by permuting the rows and relabelling the symbols. To see this consider the row bordered by $s^{i} g_{a}$. Since $N$ is normal in $G$ there is some $g_{a^{\prime}}=s^{-j} g_{a} s^{j} \in N$. Now, the entry in the column bordered by $s^{j} g_{b}$ is $s^{i} g_{a} s^{j} g_{b}=s^{i+j} s^{-j} g_{a} s^{j} g_{b}=s^{i+j} g_{a^{\prime}} g_{b}$. This means that the row of $A_{i j}$ bordered by $s_{i} g_{a}$ can be obtained from the row of $A_{11}$ bordered by $g_{a^{\prime}}$ by applying the map $x \rightarrow s^{i+j} x$. Thus $A_{i j}$ is isotopic to $A_{11}$ and must also have a 1-partition.

Arbitrarily fix an order on the transversals we have just found in each block of $L$, and choose $a$ and $b$ in the ranges $0 \leq a<\frac{1}{2} m$ and $1 \leq b \leq q$. We form a duplex $D_{a, b}$ by taking the $b$-th transversal from each of the blocks $A_{2 a+i, i}$ and $A_{2 a+1+i, i}$ for $i=0,1,2, \ldots, m-1$, where subscripts are taken modulo $m$. The blocks chosen to figure in $D_{a, b}$ correspond to a duplex of the cyclic latin square of order $m$ obtained by replacing each block in (3) by a single symbol specific to the symbols used in that block. Hence it should be clear that $D_{a, b}$ is indeed a duplex. Also, $D_{a, b}$ and $D_{c, d}$ are parallel unless $a=c$ and $b=d$, so the $D_{a, b}$ decompose $L$. This completes the proof.

With the aid of Theorem 7 and the results of Hall and Paige [17] we have resolved the existence question for plexes in soluble groups. Depending on whether such a group has a non-trivial cyclic Sylow 2-subgroup it either has a $k$-plex for all possible $k$, or has them for all possible even $k$ but no odd $k$. If the Hall-Paige conjecture could be proved it would completely resolve the existence question of plexes in groups, and these would remain the only two possibilities.

It is worth noting that other scenarios occur for latin squares which are not based on groups. For example, the following square has no transversal, but the marked entries form a 3-plex.

$$
\left(\begin{array}{llllll}
1^{*} & 2 & 3 & 4^{*} & 5 & 6^{*} \\
2^{*} & 1 & 4 & 3^{*} & 6^{*} & 5 \\
3 & 5^{*} & 1 & 6 & 2^{*} & 4^{*} \\
4 & 6 & 2^{*} & 5 & 3^{*} & 1^{*} \\
5^{*} & 4^{*} & 6^{*} & 2 & 1 & 3 \\
6 & 3^{*} & 5^{*} & 1^{*} & 4 & 2
\end{array}\right)
$$

It would be of interest to determine whether there exist arbitrarily large latin squares (presumably of even order) of this type. We conjecture that there are.

Conjecture 1. For all even $n>4$ there exists a latin square of order $n$ which has no 
transversal but does contain a 3-plex.

\section{$\S 4$. Small orders}

Finney [10-12], Freeman [13-15], and Johnston and Fullerton [19] between them have made a very detailed study of the plexes in squares of orders up to 6 . Killgrove et al. [20] studied orders 6 and 7. In this section we report, for the first time, results for order 8 squares.

We first present a table showing the range of the number of $k$-plexes (for $k=1,2,3$ ) in squares of orders 6,7 , and 8 . Since commutative group tables tend to have exceptional numbers of plexes, they have been separated in the results. We use $C(n)$ to denote the set of commutative groups of order $n$.

\begin{tabular}{|c|r|r|r|r|r|r|}
\hline & $\begin{array}{c}\text { fewest } \\
\text { 1-plexes }\end{array}$ & $\begin{array}{c}\text { most } \\
\text { 1-plexes }\end{array}$ & $\begin{array}{c}\text { fewest } \\
\text { 2-plexes }\end{array}$ & $\begin{array}{c}\text { most } \\
\text { 2-plexes }\end{array}$ & $\begin{array}{c}\text { fewest } \\
\text { 3-plexes }\end{array}$ & $\begin{array}{c}\text { most } \\
\text { 3-plexes }\end{array}$ \\
\hline $\mathrm{LS}(6) \backslash C(6)$ & 0 & 32 & 239 & 621 & 0 & 1792 \\
$C(6)$ & 0 & 0 & 1539 & 1539 & 0 & 0 \\
$\mathrm{LS}(7) \backslash C(7)$ & 3 & 63 & 2676 & 4295 & 42731 & 54288 \\
$C(7)$ & 133 & 133 & 23184 & 23184 & 310198 & 310198 \\
$\mathrm{LS}(8) \backslash C(8)$ & 0 & 384 & 23592 & 263208 & $\leq 2211280$ & $\geq 15205248$ \\
$C(8)$ & 0 & 384 & 460096 & 465976 & 0 & 28979840 \\
\hline
\end{tabular}

There are 147 main classes of order 7 latin squares (see eg. [5]). Killgrove et al. [20] reported that, except for two pairs of order 7 squares, the number of transversals together with the number of duplexes was enough to distinguish all main classes up to order 7 . It turns out that the two ties that they reported can be broken by considering the number of 3-plexes. In other words the numbers of plexes completely discriminate between all main classes up to order 7 .

There are 283657 main classes of order 8 latin square. Counting 1 and 2-plexes partitions the order 8 squares into 60224 equivalence classes, of which 26717 consist of a single main class, (which can thereby be identified by counting transversals and duplexes). Counting 3 and 4-plexes can discriminate further but is computationally expensive. The author's program took forty minutes to count the 3-plexes in a single square, and hence was too slow to establish whether all main classes could be distinguished simply on the basis of $k$-plex counts. However, it did establish that in a number of cases it will be necessary to look as far as 4-plexes. The following two squares both 
have 60 transversals, 57510 duplexes and 3620999 triplexes:

$$
\left(\begin{array}{llllllll}
1 & 8 & 6 & 4 & 7 & 3 & 5 & 2 \\
5 & 1 & 2 & 8 & 3 & 7 & 6 & 4 \\
6 & 3 & 4 & 1 & 2 & 5 & 7 & 8 \\
7 & 5 & 8 & 6 & 4 & 2 & 3 & 1 \\
4 & 7 & 5 & 2 & 8 & 6 & 1 & 3 \\
2 & 6 & 3 & 5 & 1 & 4 & 8 & 7 \\
8 & 2 & 7 & 3 & 5 & 1 & 4 & 6 \\
3 & 4 & 1 & 7 & 6 & 8 & 2 & 5
\end{array}\right) \quad\left(\begin{array}{llllllll}
4 & 7 & 8 & 1 & 3 & 2 & 5 & 6 \\
3 & 6 & 1 & 7 & 5 & 4 & 8 & 2 \\
6 & 2 & 4 & 8 & 7 & 5 & 1 & 3 \\
7 & 3 & 5 & 4 & 8 & 6 & 2 & 1 \\
8 & 4 & 7 & 3 & 2 & 1 & 6 & 5 \\
1 & 5 & 6 & 2 & 4 & 7 & 3 & 8 \\
5 & 8 & 2 & 6 & 1 & 3 & 7 & 4 \\
2 & 1 & 3 & 5 & 6 & 8 & 4 & 7
\end{array}\right)
$$

It is easily established that they are not from the same main class by, for example, counting subsquares. It seems more expedient to check such criteria than it does to establish that the square on the left has 14245160 4-plexes while the one on the right has 14250382 .

An interesting observation is that these squares have an odd number (in fact, a prime number) of 3-plexes. The significance of this remark is that Balasubramanian [1] has shown that squares of even order have an even number of transversals. It might be hoped that this result would generalise to $(2 a+1)$-plexes for $a>0$, much as the parity argument in Theorem 6 generalises an earlier result for transversals. However, our examples in (5) deny this possibility and are of the minimum order to do so. It is worth remarking further that Balasubramanian made a conjecture aimed at proving an earlier conjecture of Ryser that the number of transversals of a latin square always equals its order modulo 2. However, both conjectures fail, since there are many examples of squares of order 7 which have an even number of transversals (see [5] for examples). It seems that the result Balasubramanian proved does not generalise in any of the obvious directions.

The next table shows the squares of order $n$, for $4 \leq n \leq 8$, counted according to their maximum number of parallel transversals. That is, each main class representative $M$ is scored by the maximum $m$ such that $M$ has a $\left(1^{m}, n-m\right)$ partition. Recall that if $m=n$ then $M$ has a 1-partition and hence an orthogonal mate.

\begin{tabular}{|l|r|r|r|r|r|}
\hline$m \backslash n$ & 4 & 5 & 6 & 7 & 8 \\
\hline 0 & 1 & 0 & 6 & 0 & 33 \\
1 & 0 & 1 & 0 & 1 & 0 \\
2 & 0 & 0 & 2 & 5 & 7 \\
3 & - & 0 & 0 & 24 & 46 \\
4 & 1 & - & 4 & 68 & 712 \\
5 & - & 1 & - & 43 & 71330 \\
6 & - & - & 0 & - & 209505 \\
7 & - & - & - & 6 & - \\
8 & - & - & - & - & 2024 \\
\hline total & 2 & 2 & 12 & 147 & 283657 \\
\hline
\end{tabular}


So we see that there are 6 main classes of order 6 and 33 main classes of order 8 squares which have no transversal. There are 2024 main classes of order 8 which permit a 1-partition. Most of these are comparatively rich in transversals, but 4 of them have the bare minimum number of transversals, namely 8. One example is this square, in which subscripts denote the transversals,

$$
\left(\begin{array}{llllllll}
1_{a} & 2_{b} & 3_{c} & 4_{d} & 5_{e} & 6_{f} & 7_{g} & 8_{h} \\
7_{b} & 8_{a} & 5_{d} & 6_{c} & 2_{f} & 4_{e} & 1_{h} & 3_{g} \\
2_{c} & 1_{d} & 6_{a} & 3_{b} & 4_{g} & 5_{h} & 8_{e} & 7_{f} \\
8_{d} & 7_{c} & 4_{b} & 5_{a} & 6_{h} & 2_{g} & 3_{f} & 1_{e} \\
4_{f} & 3_{e} & 1_{g} & 2_{h} & 7_{a} & 8_{b} & 5_{c} & 6_{d} \\
6_{e} & 5_{f} & 7_{h} & 8_{g} & 1_{b} & 3_{a} & 2_{d} & 4_{c} \\
3_{h} & 6_{g} & 2_{e} & 1_{f} & 8_{c} & 7_{d} & 4_{a} & 5_{b} \\
5_{g} & 4_{h} & 8_{f} & 7_{e} & 3_{d} & 1_{c} & 6_{b} & 2_{a}
\end{array}\right)
$$

We next give the table for 3-plexes, corresponding to the one above for 1-plexes. Each square is scored by the maximum $m$ such that it has a $\left(3^{m}, n-3 m\right)$ partition, is this:

\begin{tabular}{|l|l|l|l|r|r|}
\hline$m \backslash n$ & 4 & 5 & 6 & 7 & 8 \\
\hline 0 & 1 & 0 & 4 & 0 & 1 \\
1 & 1 & 2 & - & 0 & 0 \\
2 & - & - & 8 & 147 & 283656 \\
\hline
\end{tabular}

Interestingly, up to order 8 the only squares without the maximum possible number of parallel 3-plexes are the step-type squares covered by Theorem 6 . It would be of some interest to establish for larger orders whether any other type of square can fail to have a 3-plex.

We turn finally to 2-plexes, where the results are very simple. Every square of order $n \leq 8$ contains the maximum possible number, $\lfloor n / 2\rfloor$, of parallel 2-plexes (a result previously shown by Finney $[10,11]$ for $n \leq 6)$. This suggests the following conjectures:

Conjecture 2. Every latin square of even order has a 2-partition.

Conjecture 3. Every latin square of odd order has a $(1,2,2,2, \ldots, 2)$-partition.

Note that these conjectures strengthen Rodney's conjecture [5, p.105] that every latin square has a duplex. Conjecture 3 also strengthens Ryser's conjecture that every odd order latin square has a transversal. Rodney's conjecture was proved for Cayley tables of soluble groups in [21]. We proved Conjectures 2 and 3 for the same groups in $\S 3$ above. All Cayley tables for groups of odd order have a 1-partition and hence Conjecture 3 is certainly true for them. Also, Theorem 7 proves Conjecture 2 for all groups with a cyclic Sylow 2-subgroup. It follows that this conjecture is true for all 
soluble groups, and would be established for all groups if the Hall-Paige conjecture were proved.

One obstacle to attempts to prove our conjectures are the indivisible plexes discussed next. They mean it is not reasonable to indiscriminately extract 2-plexes and expect this to lead to a 2-partition.

\section{$\S 5$. Indivisible plexes}

Say that a $k$-plex is indivisible if it contains no $c$-plex for $0<c<k$. For example, the 3-plex given in (4) is indivisible. Also, the majority of squares (at least for the small orders we studied in the preceding section) do not have a 1-partition, and it follows immediately that they must contain an indivisible $k$-plex for some $k \geq 2$. The existence of indivisible plexes is an obstacle to some obvious attacks on the conjectures we formulated in $\S 4$. In this light, the following result is bad news.

Theorem 8. For arbitrary $k$ and $n \geq k^{2}$ there exists an indivisible $k$-plex of order $n$.

Proof: Let $m=k-1$. Below we build an indivisible $k$-plex $K$ of order $k m$. The theorem will then follow easily from the following observation. If $n \geq k^{2}$ then $n-k m \geq k$ so we can find a $k$-plex of order $n-k m$ and take the direct sum with $K$ to produce a $k$-plex $K^{\prime}$ of order $n$. Any $c$-plex of $K^{\prime}$ would necessarily contain a $c$-plex of $K$, which we know does not exist if $0<c<k$.

We construct $K$ as a $k \times k$ arrangement of square blocks each of order $m$ :

$$
\left(\begin{array}{cccccc}
A_{1} & B_{1} & 0 & 0 & \cdots & 0 \\
0 & A_{2} & B_{2} & 0 & \cdots & 0 \\
0 & 0 & A_{3} & B_{3} & \cdots & 0 \\
0 & 0 & 0 & A_{4} & \cdots & 0 \\
\vdots & \vdots & \vdots & \vdots & \ddots & \vdots \\
B_{k} & 0 & 0 & 0 & \cdots & A_{k}
\end{array}\right)
$$

Each of the blocks $A_{i}$ on the main diagonal is a matrix $A$ which contains each of the symbols $1,2, \ldots, m^{2}$ exactly once (the exact position of symbols being immaterial). The blocks $B_{i}$ on the diagonal above the main diagonal each equal $B \in \operatorname{PLS}(m)$, the only filled cells of which lie on its main diagonal and are given by

$$
B_{j j}=m^{2}+j j \quad \text { for } j=1,2, \ldots, m .
$$

The remaining blocks, marked with a 0 , are all empty. It should be immediately apparent that $K$ is a $k$-plex of order $m k$. As an aside, we note that $K$ is easily completable.

Suppose that $K$ contains a $c$-plex $C$. Let $\alpha$ be the number of entries of $C$ which lie in the block $A_{1}$. Since there are $c$ entries of $C$ in each of the first $m$ rows, there 
must be $\beta=m c-\alpha$ entries of $C$ in $B_{1}$. But now counting the entries in the columns through $B_{1}$ we see that there must be $m c-\beta=\alpha$ entries of $C$ in $A_{2}$. Proceeding in this way, we ascertain that there must be $\alpha$ entries of $C$ in each $A_{i}$, and $\beta$ entries of $C$ in each $B_{i}$. Hence the total number of entries chosen from the $B_{i}$ 's is $k \beta$, and this must equal the total number of occurrences of the symbols $m^{2}+1, m^{2}+2, \ldots, m^{2}+m$ in $C$, namely $\mathrm{cm}$. But $m=k-1$ so $m$ and $k$ are relatively prime, which means the equation $k \beta=c m$ implies that $k$ divides $c$. Thus the $c$-plex that we found in $K$ can only be a trivial one; either a 0 -plex or a $k$-plex.

We make no claim as to the optimality of the bound $k^{2}$ in the theorem just proved. Another question which we leave open is the existence, for a fixed $k$, of latin squares in which every $k$-plex is indivisible. Some examples for the case $k=2$ can be found amongst the group tables discussed in $§ 3$, and (4) gives one example for $k=3$. If Conjectures 2 and 3 hold then the only examples would be of even order and we must also have $k$ odd or equal to 2 .

\section{$\S$ 6. Summary}

We have defined plexes, which are generalised transversals of latin squares. Some results about transversals (1-plexes) generalise to $k$-plexes for other $k$. The non-existence result in Theorem 6 is in this category. Other parity arguments which might be expected to behave similarly, such as Balasubramanian's theorem discussed in $\S 4$, turn out not to generalise. (We also noted in the same section the falsity of a conjecture by Balasubramanian and a related one by Ryser on the parity of the number of transversals).

The existence question for plexes in Cayley tables of finite groups was almost completely settled in $\S 3$. We also proved the existence of plexes that cannot be completed to a latin square (in $\S 2$ ) and which cannot be subdivided (in $\S 5$ ). In $\S 4$ we gave results on plexes in squares of orders up to 8 , and conjectured that all squares have orthogonal partitions of a particular type. In doing so we strengthened Ryser's conjecture that every latin square has a transversal. Two other directions for future research that we left open are (1) A conjecture that there exist arbitrarily large even order squares with a 3-plex but no transversal and (2) a question as to whether there are squares without 3 -plexes other than those covered by Theorem 6 .

\section{References.}

[1] K. Balasubramanian, On transversals in latin squares, Linear Algebra Appl. 131 (1990), 125-129.

[2] R. A. Bailey, Orthogonal partitions in designed experiments (Corrected reprint), Des. Codes Cryptogr. 8 (1996), 45-77.

[3] B. Burton, Completion of partial latin squares, honours thesis, University of Queensland, 1997. 
[4] A. Chetwynd and R. Häggkvist, Completing partial $n \times n$ latin squares where each row, column and symbol is used at most $c n$ times, unpublished.

[5] C. J. Colbourn and J. H. Dinitz (eds) The CRC handbook of combinatorial designs, CRC Press, Boca Raton, FL, 1996.

[6] D. E. Daykin and R. Häggkvist, Completion of sparse partial latin squares, Graph theory and combinatorics, 127-132, Academic Press, London, 1984.

[7] J. Dénes and A. D. Keedwell, Latin squares and their applications, Akadémiai Kiadó, Budapest, 1974.

[8] J. Dénes and A. D. Keedwell, Latin squares: New developments in the theory and applications, Annals Discrete Math. 46, North-Holland, Amsterdam, 1991.

[9] D. Donovan, The completion of partial latin squares, Australas. J. Combin. 22 (2000), 247-264.

[10] D. J. Finney, Some orthogonal properties of the $4 \times 4$ and $6 \times 6$ latin squares. Ann. Eugenics 12 (1945), 213-219.

[11] D. J. Finney, Orthogonal partitions of the $5 \times 5$ latin squares. Ann. Eugenics 13 (1946), 1-3.

[12] D. J. Finney, Orthogonal partitions of the $6 \times 6$ latin squares. Ann. Eugenics 13 (1946), 184-196.

[13] G. H. Freeman, Duplexes of $4 \times 4,5 \times 5$ and $6 \times 6$ latin squares. Utilitas Math. 27 (1985), 5-24.

[14] G. H. Freeman, Triplex properties of $6 \times 6$ latin squares. Utilitas Math. 41 (1992), 11-32.

[15] G. H. Freeman, Orthogonal partitions of $6 \times 6$ latin squares of Set X. Utilitas Math. 41 (1992), 139-149.

[16] D. C. Gilliland, A note on orthogonal partitions and some well-known structures in design of experiments, Ann. Statist. 5 (1977), 565-570.

[17] M. Hall and L. J. Paige, Complete mappings of finite groups, Pacific J. Math. 5 (1955), 541-549.

[18] P. Horák and A. Rosa, Maximal partial latin squares, Lecture Notes in Pure and Appl. Math., 139 (1993), 225-235.

[19] B. Johnston and T. Fullerton, Construction of the $3^{2}$ orthogonal partitions of $6 \times 6$ latin squares, Congr. Numer. 42 (1984), 199-212.

[20] R. Killgrove, C. Roberts, R. Sternfeld, R. Tamez, R. Derby and D. Kiel, Latin squares and other configurations, Congr. Numer. 117 (1996), 161-174.

[21] M. Vaughan-Lee and I. M. Wanless, Latin squares and the Hall-Paige conjecture, Bull. London Math. Soc., (to appear). 